\title{
A Atuação das Comissões Intergestores Bipartite de Minas Gerais e Rio de Janeiro frente às necessidades de Saúde (2012-2013)
}

The Performance of the Intermanagers Bipartite Commissions of the State of Minas Gerais and Rio de Janeiro in face of the necessities of Health (2012-2013)

El funcionamiento de las Comisiones Intergestores bipartidas en los Estados de Minas Gerais y Rio de Janeiro delante las necesidades de la salud (2012-2013)

Mayara Nepomuceno Corrêa dos Santos ${ }^{1}$ Ivan Pricken de Bem²

RESUMO: O artigo tem como objetivo verificar a atuação das Comissões Intergestores Bipartite (CIB) de Minas Gerais e do Rio de Janeiro frente as especificidades de saúde locais nos anos de 2012 e 2013. Tratou-se de uma pesquisa qualiquantitativa com análise documental das Resoluções das Comissões Intergestores Bipartite dos estados disponíveis na internet por meio dos respectivos sítios eletrônicos. Os estados possuem especificidades locais distintas, sendo que os programas estaduais voltados para a Saúde Indígena foram o destaque de Minas Gerais e as ações voltadas para a melhoria da Atenção Especializada foi o destaque do Rio de Janeiro. As Comissões Intergestores Birpatite têm desempenhado um papel fundamental na formulação de políticas públicas visando a melhoria de saúde da população, de acordo com os princípios do Sistema Único de Saúde (SUS).

Palavras-chave: Comissão Intergestores Bipartite. Sistema de saúde. Legislação Sanitária.

ABSTRACT: The article had as objective to verify the performance of Bipartite Commissions (CIB) of the State of Minas Gerais and Rio de Janeiro about the local health specialties in the years of 2012 and 2013. It was a qualiquantitativa research with documentary analysis of the resolutions of the commissions available in their electronic sites. Results: Both states possess distinct local specialties. The indigenous health program was the mostly prominence in the State of Minas Gerais and the specialized health attention was the most viewed in the State of Rio de Janeiro. The CIB of both states has played a basic role in the formularization of public politics in accordance with the principles of the Unified Health System (SUS).

Keywords: Unified Health System (SUS). Intermanager Bipartite Commissions. Health Legislation.

RESUMEN: El artículo tiene el objetivo de hacer una verificación en el funcionamiento de las Comisiones Intergestores bipartidas del Estado de Minas Gerais y Río de Janeiro, sus especialidades locales en la salud en los años de 2012 y 2013. Hizo una investigación cualitativa y cuantitativa por medio del análisis de las resoluciones de las Comisiones Intergestores bipartidas (CIB) de los estados disponibles en respectivos sitios electrónicos.

\footnotetext{
${ }^{1}$ Estudante de graduação de Gestão em Saúde Coletiva, Departamento de Saúde Coletiva - Universidade de Brasília,mayncs@hotmail.com

2 Pesquisador Colaborador, Fundação Oswaldo Cruz - Brasília, ivanprk@gmail.com
} 
Resultados: Los estados poseen especificidades locales distintos. Sobre la salud indígena tienen más prominencia en el estado de Minas Gerais y las acciones de la atención especializada en salud tuvieron más prominencia en Río de Janeiro. Las CIB han desempeñado un papel básico en la formulación de las políticas públicas de salud para la población, de acuerdo con los principios del sistema de salud (SUS).

Palabra-Ilave: Comisión Intergestores bipartidas. Sistema de la salud. Legislación Sanitaria.

\section{Introdução}

A criação do Sistema Único de Saúde (SUS) é consequência da luta do Movimento pela Reforma Sanitária, um grande marco histórico para saúde brasileira. Este processo teve como marco institucional a 8ª Conferência Nacional de Saúde, realizada em 1986 em que através desse movimento a população começou a reivindicar melhores condições de saúde e a criação de um sistema capaz de atender às suas necessidades de forma gratuita e igualitária. O relatório final deste marco da saúde pública brasileira, serviu de base para a o artigo 196 da constituição, que tornou a saúde como direito universal calcado no princípio da dignidade da pessoa humana (1).

Durante o processo de fortalecimento do SUS ocorreram diversas mudanças na legislação brasileira com o objetivo de acompanhar as mudanças sociais e econômicas no Brasil. O Conselho Nacional de Saúde (CNS) teve grande importância no processo de consolidação desse sistema por ser um órgão deliberativo responsável pelas bases gerais dos programas de proteção à saúde (2). Com o objetivo de auxiliar na implantação e operacionalização regional do SUS junto com o CNS, nascem as Comissões Intergestores Bipartite (CIB), uma estrutura deliberativa instituída gradativamente nos estados brasileiros durante os anos de 1993 e 1994, a partir de determinação da Portaria do Ministério da Saúde 545 de 20 maio de 1993, que estabeleceu a Norma Operacional Básica SUS (NOB SUS) $01 / 93$.

Essas comissões são compostas igualitariamente por dirigentes da Secretaria de Saúde do Estado e dos órgãos de representação estadual dos Secretários Municipais de Saúde (COSEMS) responsáveis pela formulação de Políticas Públicas estaduais visando a melhoria da saúde da população de acordo com os princípios do Sistema Único de Saúde.

Para Viana e Lima (3):

Essa nova estrutura colegiada, acrescentado à estrutura de gestão do SUS no plano estadual visa mudar a estratégia política de descentralização das 
ações e serviços de saúde, de forma a fortalecer o papel regulador dos estados e a criação de sistemas supra municipais. Assim, às CIB foi atribuído o papel de adequar as normas nacionais às condições específicas de cada estado e propor medidas reguladoras das relações estado/municípios e município/município na implementação do SUS.

Na região Sudeste, as Comissões foram implementadas no ano de 1993, sendo no estado do Rio de Janeiro criada através da Resolução estadual no 855/1993. De acordo com a Secretaria de Saúde do estado as reuniões da CIB/RJ só começaram a ocorrer de forma regular a partir de 1994 devido à falta de nomeação dos membros suplentes que iriam participar da comissão. Nesse contexto o Ministério da Saúde teve que intervir diretamente devido ás resistências inicias dos membros da Secretaria de Estado (4).

Para os municípios essa implantação era uma forma de participação direta no processo de descentralização do SUS. No Estado de Minas Gerais (CIB-SUS/MG) esta instância colegiada foi instituída pela Resolução estadual no 637 em 1993, com a finalidade de regulamentar e aperfeiçoar aspectos operacionais do SUS/MG. Mesmo sendo o estado brasileiro com maior número de municípios, totalizando 853, a CIB/MG possui uma comissão menor que o Rio de Janeiro, ambas sendo divididas de forma igualitária entre a Secretaria de Saúde do Estado e os Secretários Municipais de Saúde.

Os diferentes contextos vivenciados nas regiões do Brasil exigem que sejam desenhadas políticas capazes de atender a necessidade de cada população, com isso, o papel das comissões é fundamental para a descentralização das ações e serviços de saúde nos Estados e organização do sistema de saúde estadual, incentivando a responsabilização dos municípios no exercício de suas funções gestoras e fortalecimento do controle social.

Neste contexto, o presente artigo tem como objetivo verificar a atuação das Comissões Intergestores Bipartite de Minas Gerais e do Rio de Janeiro frente as especificidades de saúde locais nos anos de 2012 e 2013.

\section{Metodologia}

Foi realizada uma pesquisa qualiquantitativa por meio da análise documental das Resoluções das Comissões Intergestores Bipartite de 2012 e 2013 dos Estados de Minas 
Gerais e Rio de Janeiro disponíveis na internet por meio dos sítios eletrônicos ${ }^{3}$ http://www.saude.mg.gov.br/ e www.cib.rj.gov.br/deliberacoes-cib, respectivamente.

As resoluções foram lidas e classificadas de acordo a abrangência de seu conteúdo. As 25 categorias de classificação das resoluções que englobam assuntos desde a gestão até a atenção básica foram elaboradas pelos autores no propósito de abranger o objeto da saúde e investimentos no setor.

Por meio da categorização, foi realizado uma análise comparativa com os dados obtidos através do estudo, para apontar quais são as principais especificidades e melhorias dos estados.

Os dados foram sistematizados em planilhas Microsoft Excel 2008 para posteriormente gerar dados e análises mais profundas em consonância com as necessidades de saúde dos Estados brasileiros do estudo.

\section{Resultados e Discussão}

Para a realização deste trabalho foram analisadas 1.850 resoluções, dessas 1.174 correspondem ao estado do Rio de Janeiro e 678 à Minas Gerais. Das análises, foram excluídas 60 resoluções da CIB por não estarem disponibilizadas ao público no site oficial de seus órgãos.

Tabela 1. Resolução das Comissões Intergestoras Birpatite de Minas Gerais por assunto (2012-2013)

\begin{tabular}{lll}
\hline Categorias & 2012 & 2013 \\
\hline Gestão, programas e planos & 117 & 144 \\
Repasse e Financiamento & 100 & 123 \\
Atenção Básica & 50 & 51 \\
Outros & 31 & 57 \\
Redes de Atenção à Saúde & 39 & 47 \\
Hospitais e outras unidades de saúde & 57 & 27 \\
Saúde Bucal & 39 & 36 \\
Leitos & 32 & 39 \\
Vigilância em Saúde & 32 & 29 \\
Convênio Médico e SUS & 23 & 35 \\
Procedimento e Tratamento médico ou hospitalar & 27 & 18 \\
Educação e Trabalho em Saúde & 24 & 21
\end{tabular}

\footnotetext{
${ }^{3}$ http://www.saude.mg.gov.br/sobre/institucional/deliberacao-cib-susmg
} 


$\begin{array}{lll}\text { Saúde Indígena } & 14 & 8 \\ \text { Criança e Adolescente } & 10 & 23 \\ \text { Saúde Mental } & 12 & 15 \\ \text { Medicamentos e Insumos } & 11 & 14 \\ \text { Mulher } & 6 & 16 \\ \text { Equipamentos e Materiais para Saúde } & 11 & 11 \\ \text { Doenças Crônicas e Não Transmissíveis } & 8 & 10 \\ \text { Álcool, Tabaco e outras drogas } & 8 & 7 \\ \text { Doenças Infecciosas e Parasitárias } & 12 & 3 \\ \text { Idoso } & 2 & 8 \\ \text { Alimentação e Nutrição } & 4 & 3 \\ \text { Sangue, Transplante de órgãos e células } & 1 & 0 \\ \text { DST/AIDS } & 1 & 0 \\ \text { Saúde do Trabalhador } & 0 & 0\end{array}$

Fonte: Elaborada com base nos dados obtidos no portal da CIB Minas Gerais

Observou-se que das 25 classificações 4 se destacam por sua frequência no estado em contraste aos demais: Gestão, programas e planos, Repasse e Financiamento, Atenção Básica e Redes de Atenção à Saúde. Essas categorias se sobressaíram em Minas Gerais porque através da análise das resoluções é possível notar um investimento no repasse de recursos para os programas estaduais/locais voltados principalmente para a atenção primária em saúde.

O Pro-Hosp foi um programa instituído pelo governo do Estado de Minas Gerais em 2003, com o objetivo de promover a melhoria da qualidade dos hospitais integrantes do Sistema Único de Saúde (5) assegurando o atendimento de qualidade para a população mineira, de forma que o usuário não tenha que se deslocar frequentemente de sua residência para uma consulta médica. Nesse cenário o atendimento nos hospitais é realizado próximo ao domicílio do usuário. Isso se deve a parceria público privada com os hospitais filantrópicos responsáveis por garantir esse atendimento. O repasse de verbas é feito de acordo com a realidade local de cada município com ampla cobertura nas 75 microrregiões do estado.

O programa Saúde em Casa se destacou nas resoluções classificadas como Atenção Básica por ser uma política estruturadora voltada para a universalização do acesso e melhoria do serviço da atenção primária no estado garantindo o atendimento de qualidade em domicílio para os usuários (6).

As deliberações sobre Redes de Atenção à Saúde versaram principalmente na saúde da pessoa com necessidades especiais, destacando a importância da Rede Estadual de 
Saúde Auditiva que garante a inclusão do paciente com deficiência no SUS e a Rede Cegonha que através da resolução № 1.681 de 2013 teve garantida a sua expansão assegurando maiores quantidades de pontos de atenção referentes a saúde maternoinfantil no estado.

Resoluções sobre a Saúde Indígena foi o ponto forte em Minas Gerais, pois diferentemente da outra unidade da federação analisada, Minas Gerais possui um forte investimento nessas políticas que resgatam a cultura dos povos indígenas e fortalecem os seus laços com o SUS (7).

Para assegurar o direito a saúde para toda a população, o governo mineiro elaborou a Política Estadual de Saúde Indígena que garante para as 10 etnias, residentes em aldeias e reservas de 15 municípios, o direito de ter políticas públicas capazes de levar melhoria nas condições de saúde da população.

Para Passos (7):

A Política Estadual de Saúde Indígena é dividida em eixos de atuação em diversos programas com ações de saúde mental, resgate e manutenção do conhecimento indígena em sua medicina tradicional, oficinas e troca de experiências para garantir uma cobertura ampla e diversa.

Através da criação dessas ações, foi implantada a Coordenadoria Estadual de Saúde Indígena (CESI) responsável por assegurar e fiscalizar os serviços prestados aos indígenas e são na Atenção Básica as principais ações realizadas para a melhoria da saúde dessa população.

Um exemplo de programa estadual eficaz nos municípios é o de Saúde Mental Indígena, devido ao uso frequente dessa população de álcool e tabaco nas cerimonias ritualísticas. Esse programa nasceu com o "objetivo reduzir o uso de álcool, um dos principais agravos à saúde dos povos indígenas, e também os índices de depressão e suicídio" (7). Nessas ações os Agentes Indígenas de Saúde (ASI) promovem ações de promoção em saúde voltadas para a redução dos agravos relacionados ao uso desenfreado dessas substâncias.

A população indígena, mesmo contando com poucos dados epidemiológicos relacionados ao uso de drogas apresenta estudos importantes para a elaboração de políticas sociais como o da Fundação Nacional de Saúde (FUNASA) que constatou que uso abusivo de álcool é um dos principais agravos na saúde das populações indígenas 
brasileiras sendo mais frequente a dependência nas aldeias próximas das cidades por ser mais fácil o comércio de destilados (8).

Tabela 2. Resolução das Comissões Intergestoras Birpatite do Rio de Janeiro por assunto (2012-2013)

\begin{tabular}{lll}
\hline Categorias & 2012 & 2013 \\
\hline Hospitais e outras unidades de saúde & 96 & 179 \\
Gestão, programas e planos & 116 & 150 \\
Atenção Básica & 47 & 180 \\
Equipamentos e Materiais para Saúde & 96 & 78 \\
Outros & 32 & 110 \\
Redes de Atenção à Saúde & 49 & 61 \\
Leitos & 47 & 43 \\
Convênio Médico e SUS & 43 & 32 \\
Procedimento e Tratamento médico ou hospitalar & 50 & 22 \\
Vigilância em Saúde & 32 & 30 \\
Saúde Mental & 50 & 6 \\
Saúde Bucal & 33 & 23 \\
Repasse e Financiamento & 38 & 15 \\
Educação e Trabalho em Saúde & 26 & 26 \\
Álcool, Tabaco e outras drogas & 27 & 22 \\
Criança e Adolescente & 13 & 13 \\
Doenças Crônicas e Não Transmissíveis & 6 & 8 \\
DST/AIDS & 10 & 3 \\
Sangue, Transplante de órgãos e células & 4 & 6 \\
Mulher & 3 & 7 \\
Doenças Infecciosas e Parasitárias & 5 & 4 \\
Medicamentos e Insumos & 5 & 1 \\
Alimentação e Nutrição & 0 & 1 \\
Saúde do Trabalhador & 0 & 1 \\
Idoso & 0 & 0 \\
\hline
\end{tabular}

Fonte: Elaborado com base nas informações do portal da CIB do Rio de Janeiro

No estado do Rio de Janeiro, as quatro classificações que se destacaram foram: Hospitais e outras unidades de saúde, Gestão, programas e planos, Saúde Mental e Atenção Especializada. Ao contrário de Minas Gerais em que o foco é a atenção básica, o estado do Rio de Janeiro possui uma maior demanda no atendimento de urgência e emergência, tendo a maioria das suas resoluções classificadas como Hospitais e outras 
Unidades de Saúde voltadas para a construção de Unidades de Pronto Atendimento (UPA) e Unidades Básicas de Saúde (UBS). Além disso, foi possível notar que o estado possui uma ampla cobertura na populacional de atenção integral e contínua para pessoas com transtorno psicossocial ou necessidades decorrentes do uso de álcool, tabaco e outras drogas, sendo possível destacar o programa de controle ao tabagismo que apareceu em 19,25\% das deliberações classificadas como gestão programas e planos e saúde mental.

Essa política tem por finalidade a diminuição do consumo de tabaco da população e oferecer tratamento para os usuários que desejam se livrar do vício. Foi possível notar através das resoluções que para solucionar o problema com álcool, tabaco e outras drogas o estado investe também na construção de Centros de Atenção Psicossocial (CAPS), principalmente em CAPS I que tem por finalidade o cuidado de adultos com transtornos mentais e possui um grande papel na busca da reintegração desses usuários com a sociedade (9).

O grande destaque do estado foi para a categoria Outros que apareceu em 9,36\% das deliberações. Dentro dessa categoria, parte das resoluções são relacionadas a Atenção Especializada sendo que o Estado busca investir no programa federal Tratamento Fora de Domicílio (TFD) que tem por finalidade atender os pacientes de doenças não tratáveis em seu município e deslocá-lo para o atendimento na região de cobertura mais próxima com ajuda de custo governamental (10).

O Rio de Janeiro possui grande repasse destinado a atenção especializada, principalmente para aquisição de equipamentos e materiais para saúde (11). Esta categoria apareceu com $14,82 \%$ nas deliberações destinadas a financiar a compra de equipamentos ao referido nível de atenção assim como para a construção de hospitais para o atendimento de média e alta complexidade, aumentando assim a cobertura da Rede de Urgência e Emergência do estado.

Durante a análise comparativa dos dados foi possível destacar quatro categorias. A categoria Repasse e Financiamento apareceu $28 \%$ a mais do que no estado de Minas Gerais, principalmente para a destinação de recursos aos programas estaduais de atenção primária. No Rio de Janeiro ocorreu diminuição de repasse para a saúde principalmente no ano de 2013. Na categoria Atenção Básica houve um aumento de $20 \%$ no estado do Rio de Janeiro, sendo possível verificar que ocorreu uma ampliação na cobertura da Estratégia Saúde da Família, trazendo avanços no atendimento da atenção primária, principalmente 
na atenção domiciliar, sendo nesse mesmo ano implementado o serviço de Atenção Domiciliar para a população idosa do estado (11).

Através desse estudo foi possível notar que o estado de Minas Gerais aumentou suas resoluções voltadas para a Saúde Mental. A classificação nessa categoria ocorreu principalmente nas deliberações voltadas para criação de Centros de atenção Psicossocial (CAPS) nos municípios e um maior investimento do governo nessa área mostrando um aumento principalmente em 2013. Um ponto que merece destaque nessa categoria é a queda de $7 \%$ no estado do Rio de Janeiro nesse mesmo ano, mostrando que ocorreu uma mudança nas necessidades de saúde de sua população.

Outra categoria que se destacou, foi a destinada a criação de Hospitais e outras unidades de saúde em que o estado do Rio de Janeiro aumentou principalmente nas resoluções destinadas a criação de unidades básicas de saúde para a atenção básica. $\mathrm{O}$ estado de Minas Gerais nessa categoria apresentou uma queda mostrando que as principais deliberações são voltadas para a criação de unidades de pronto atendimento (UPA) para a melhoria do atendimento de urgência e emergência do estado. As outras categorias não tiveram alterações visíveis e com isso não foram comparadas nesse estudo.

\section{Considerações Finais}

No decorrer do estudo foi possível notar que ambos os estados apresentam especificidades locais distintas. Ao mesmo tempo que eles possuem maior investimento em programas que necessitam de maior prioridade pela demanda, não deixam de focar em outros tipos de atenção, como o exemplo da Primária e Especializada. Através desse estudo é possível destacar as ações propostas pelo estado de Minas Gerais na Saúde Indígena, sendo possível notar através das resoluções o interesse do estado em garantir a essa população o acesso ao Sistema Único de Saúde de forma que sejam desenhadas políticas e programas capazes de atender as suas necessidades de saúde.

As Comissões Intergestores Birpatite junto com os Conselhos de Saúde formam uma das principais instâncias colegiadas do SUS e assumem um elevado grau de importância na formulação e implementação da política de saúde no Brasil. Portanto, o Conselho de Saúde tem um importante papel na busca da participação e controle social, sendo passado a CIB a atribuição de formular resoluções que preencham a demanda de saúde da 
população, tendo como principal desafio para que seja realizada uma gestão eficaz a parceria entre a secretaria de saúde do Estado e Município. Através dessa pesquisa, os dados obtidos poderão servir de orientação para as Comissões dos estados na formulação de novas políticas.

\section{Referências}

1 Costa MI, Lapeta A. Avanços e desafios na municipalização do Sistema Único de Saúde no Brasil. In: XVII Congreso Internacional del CLAD sobre la Reforma del Estado y de la Administración Pública, 30 oct - 02 nov 2012, Cartagena, Colombia.

2 Ribeiro JM. Conselhos de saúde, comissões intergestores e grupos de interesses no Sistema Único de Saúde (SUS). Cadernos de Saúde Pública. 1997, 13 (1): 81-92 [Acesso em 28 nov. 2015]. Disponível em http://www.scielo.br/pdf/csp/v13n1/0226.pdf

3 Viana AL, Lima LD. [Relatório de Projeto]. Avaliação Nacional das Comissões Intergestores Bipartite (CIBs). Brasília: Ministério da Saúde; 2007.

4 Silva IF, Labra ME. As instâncias colegiadas do SUS no Estado do Rio de Janeiro e o processo decisório. Caderno de Saúde Pública. 2001, 17 (1): 161-170. [Acesso em 28 dez 2015]. Disponível em http://www.scielosp.org/pdf/csp/v17n1/4072.pdf

5 Secretaria de Saúde. Programa Saúde em Casa. Minas Gerais, 2015. [Acesso em 20 dez 2015]. Disponível em http://www.saude.mg.gov.br/cidadao/programas/program/12saude-em-casa

6 Fontes FB, Veiga L, Pereira DR, Camargos MC. Desafios à Implementação de Políticas Sociais: Discussões Acerca da Avaliação do Programa de Fortalecimento e Melhoria da Qualidade dos Hospitais do SUS-MG (PRO-HOSP). Revista Gestão \& Tecnologia. 2003, 13 (1): 127-150. [Acesso em 11 dez 2016]. Disponível em

http://www.repositorio.fip.mg.gov.br/bitstream/123456789/59/1/Desafios\%20\%C3\%A0\%201 mplementa\%C3\%A7\%C3\%A30\%20de\%20Pol\%C3\%ADticas\%20Sociais.pdf ]

7 Passos A. Povos Indígenas recebem atenção especial em relação a saúde. Minas Gerais, 2012. [Acesso em 10 nov 2015]. Disponível em http://www.saude.mg.gov.br/gripe/story/6940-povos-indigenas-de-minas-recebem-atencaoespecial-em-relacao-a-saude

8 Ministério da Justiça (BR), Secretaria Nacional de Políticas sobre Drogas. Sistema para detecção do uso abusivo e dependência de substâncias psicoativas: encaminhamento, intervenção breve, reinserção social e acompanhamento. Brasília: Ministério da Justiça, 2014. [Acesso em 01 fev 2016]. Disponível em:

www.supera.senad.gov.br/mod/resource/view.php?id=965 
9 Onocko-Campos RT; Furtado JP. Entre a saúde coletiva e a saúde mental: um instrumental metodológico para avaliação da rede de Centros de Atenção Psicossocial (CAPS) do Sistema Único de Saúde. Caderno de Saúde Pública. 2006, 22 (5): 1053-1062. [Acesso em 10 de abr 2016]. Disponível em http://www.scielo.br/scielo.php?script=sci arttext\&pid=S0102311X2006000500018\&lng=en\&nrm=iso

10 Rohde EB, Dobashi BF, Oliva CA. A regulação do acesso interestadual de pacientes no Sistema Único de Saúde. Cadernos Saúde Coletiva. 2009, 17 (4): 1031 - 1046. [Acesso em 10 de abr 2016]. Disponível em http://www.iesc.ufrj.br/cadernos/images/csc/2009 4/artigos/Artigo 7.pdf

11 Secretaria de Estado do Rio de Janeiro. [Relatório de Gestão]. Relatório de gestão período de janeiro a dezembro de 2013. Rio de Janeiro: Secretaria de Saúde; 2013.

Recebido em: 12/12/2015 Reapresentado em: 15/04/2016 Aprovado em: 29/08/2016

\section{Como citar este artigo:}

Santos MNC. A Atuação das Comissões Intergestores Bipartite de Minas Gerais e Rio de Janeiro frente às necessidades de Saúde (2012-2013). Revista Cadernos Ibero-Americanos de Direito Sanitário. 2016 out./dez, 5(4):112-122. 